\title{
LITERATURA POPULAR IMPRESA Y PROYECCIÓN SOCIAL: PLIEGOS POÉTICOS DEL SIGLO XVI CON DEDICATARIO
}

\author{
LAURA PUERTO MORO \\ Universidad Complutense de Madrid - IUMP
}

Resumen

Este trabajo se propone analizar un conjunto de pliegos poéticos del s. XVI muy apartado de los cauces más idiosincráticos de la literatura de cordel, el que aúna texto literario y proyección social a través de la inclusión de un dedicatario de alto rango. El artículo fija el corpus de pliegos poéticos quinientistas con dedicatario, aísla su particular codificación editorial y se adentra en algunas de las temáticas más representativas dentro de ese corpus, especialmente en la religiosa. La atención al número de dedicatarias femeninas y su comparación porcentual con trabajos previos sobre la presencia de dedicatarias en libros de los Siglos de Oro arroja interesantes datos relativos a la mayor presencia de la mujer en estos impresos menores, en particular en relación con el opúsculo religioso. Finalmente, y al hilo de tres pliegos muy particulares, se reflexiona sobre el papel que estos impresos pudieron cumplir como promoción y publicidad para una determinada obra.

Palabras clave: pliegos poéticos, siglo XVI, dedicatarios, proyección social, publicidad.

\section{PRINTED POPULAR LITERATURE AND SOCIAL PROJECTION: SIXTEENTH CEN- TURY CHAPBOOKS IN VERSE WITH DEDICATEE}

\begin{abstract}
This work sets out to scrutinize a set of XVI Century chapbooks in verse that are divergent from the most idiosyncratic lines of the Spanish literature of colportage. The analyzed chapbooks are pieces where literary text and social projection are merged by means of a dedication to a high rank person. So, the goals are first, to specify the corpus of XVI chapbooks in verse with dedicatee, second, to determine its particular editorial form and third, to delve into the most representative subject matters, specially the religious one. The attention to the number of female dedicatees and its comparison with previous works on the presence of female dedicatees in books written in the Golden Centuries yield interesting data as to the higher presence of women in these minor prints, in
\end{abstract}


particular with respect to little religious treatises. Finally, and as regards to three very particular chapbooks, I reflect on the role that these prints may have played as promotion and publicity for a given book.

Keywords: chapbooks in verse, Sixteenth century, dedicatees, social projection, publicity.

Frente a la tradicional aprehensión historiográfica del corpus de pliegos sueltos poéticos del s. XVI como un conjunto uniforme -al menos desde un punto de vista material-y esencialmente vinculado con «lo popular», el enorme avance de los últimos tiempos sobre esta parcela investigadora está comenzando a poner de manifiesto su carácter fundamentalmente heterogéneo, y ello a todos los niveles: desde la diversidad de temáticas y codificaciones editoriales que las acompañan, hasta lo falaz de la identificación global de esos impresos con una literatura popular, al menos en el sentido más estigmatizado del adjetivo.

Siguiendo por esa vereda, el presente estudio pretende ahondar en un conjunto de pliegos poéticos del siglo XVI muy particular, el que aúna texto literario y proyección social a través de la inclusión de un dedicatario de alto rango, elemento que ya en 1973 García de Enterría (1973: 119) consideraba como propio de un tipo de "pliego culto» -según su aprehensión terminológica- muy alejado de lo que ella misma denomina «los pliegos de cordel auténticos» ${ }^{1}$. Si bien la diferenciación no nos parece tan tajante, comenzando porque, tal y como veremos, en ocasiones un pliego publicado bajo la búsqueda del prestigio de la dedicatoria llega a «popularizarse» y a perder ese inicial rasgo distintivo, lo cierto es que el grueso del corpus que ahora nos ocupa constituye un subconjunto muy idiosincrático y peculiar entre los pliegos poéticos del siglo XVI, ello, desde el predominio de codificaciones editoriales muy reconocibles hasta la predilección por determinadas temáticas, siempre con la búsqueda del engaste del discurso literario en el discurso social como eje vertebrador.

Tras una revisión muy exhaustiva del Diccionario bibliográfico de pliegos sueltos poéticos del s. XVI, de Rodríguez-Moñino (1970), en su ampliación por Askins e Infantes (Rodríguez-Moniño, Askins e Infantes,

${ }^{1}$ La acotación cronológica nos lleva a dejar a un lado los particulares pliegos incunables, cuya estrecha vinculación con el mecenazgo fue ya bien estudiada en un artículo clásico de Infantes (1989) sobre la materia. 
1997), más su Suplemento (Askins e Infantes, 2014), es decir, sobre un catálogo de más de 1500 ítems, hemos podido aislar hasta cuarenta y dos pliegos -entendiendo como pliego una unidad textual ${ }^{2}$ - cuyo dedicatario pertenece a la realeza, a la esfera nobiliaria o eclesiástica, o se vincula con un cargo civil destacado, dejando a un lado, por tanto, todos aquellos textos dirigidos indeterminadamente a "un amigo», «una dama», «una señora», etc., pues creemos que poco aportan desde la óptica de imbricación impreso-proyección social que aquí nos interesa ${ }^{3}$. Entre estos cuarenta y dos pliegos, merecerán comentario aparte los impresos de tipo noticiero-celebrativo que conectan en alguna medida con la epístola de nuevas y su intrínseco destinatario, si bien hemos omitido de nuestro corpus, por razones obvias, los que se adhieren a la tipología específica de "traslado de una carta», de la misma manera que no consideramos otras obras con destinatario que, a todas luces, responden a tradiciones propias y divergentes de la aquí contemplada ${ }^{4}$. Junto con todo ello, cabe

${ }^{2}$ El paréntesis aclaratorio sobre la consideración del término "pliego» como unidad textual y no editorial en el cómputo ofrecido se vincula con el hecho de que de varios de los pliegos contemplados conservamos sucesivas ediciones.

${ }^{3}$ En este sentido, el corpus acotado se diferencia de manera muy considerable del contemplado por Di Stefano (2014) en su acercamiento a los pliegos sueltos poéticos del s. XVI con destinatario, de la misma manera que difieren ampliamente la metodología de análisis y focos de atención considerados. Dejamos constancia de que la atención a la indeterminación del dedicatario como criterio estricto para la exclusión de un ítem nos lleva, por ejemplo, a dejar fuera -con dudas- opúsculo tan ligado a los nuestros en sus características formales y materiales como es RM 414. Imitación del planto de Jeremías (1534), del Bachiller Pacheco, contrafactum profano cuyo prólogo en prosa se dirige a una «muy reverenda señora» anónima. Bajo las siglas RM remitimos siempre al número de registro del pliego en Rodríguez-Moñino (1970), en su ampliación (Rodríguez-Moñino, Askins e Infantes, 1997), más su Suplemento (Askins e Infantes, 2014).

${ }^{4}$ De esta manera, quedan fuera del corpus pliegos de trasfondo epistolar como RM 950.5, «Nuevas de Italia venidas de Boloña a Madrid: sábado xv de enero: año de mil y quinientos y treinta [...] escriviolas el mayordomo mayor de su magestad a la señora doña Inés Manrique» (1530); o RM 1092.3, «Traslado de la carta que embió su Real magestad de la emperatriz nuestra señora al muy illustrísimo seño [sic] Cardenal y al muy illustre cabildo desta muy nobe [sic] e muy leal ciudad de Sevilla. En la qual haze saber la desseada vitoria contra Barbarroja y de la ciudad de Tunes» (1535). De la misma forma, desconsideramos los pliegos ligados con ejercicios universitarios celebrativos del tipo de RM 125.5, "Al doctor Juan Ibáñez [...] habiendo llevado la Cáthedra de Prima de Cánones en la insigne Universidad de Salamanca, y solenizando el Triumpho della, de Frey Miguel Zejudo [...]» (1597); al igual que omitimos aquellos pliegos cuya presentación responde a la transcripción de una rúbrica cancioneril, tal RM 460, «Metáfora en metros que fizo Quiros al señor Juan Fernández d'Eredia siendo servidor de la señora Gerónima Beneyta [...]» (c. 1515). Nos parece improcedente, asimismo, la inclusión de un pliego poético tan particular como 
señalar que trataremos de forma diferenciada, por su especificidad, un pliego burlesco cuyo tono paródico se extiende a la dedicatoria y tres «pliegos» muy particulares, vinculados con la transmisión exenta de la dedicatoria de una obra mayor ${ }^{5}$.

Al margen de estos cuatro últimos impresos, entre los treinta y ocho restantes hay que destacar, para iniciar el análisis, la ingente presencia de pliegos de carácter religioso -hasta un $42.1 \%$ del corpus, concretamente, dieciséis-, temática por antonomasia, por otra parte, de las obras áureas con dedicatario femenino, una circunstancia en la que han insistido recientes estudios como los de Nieves Baranda (2011), o Collantes y García Aguilar (2015). Al lado de esos pliegos religiosos, detendremos nuestra atención en un subgrupo característico y hasta cierto punto homogéneo dentro del conjunto, el correspondiente a la narración de entradas reales en una ciudad, que alcanza a cuatro de los cinco pliegos noticieros incluidos en el corpus; materias a las que habría que sumar temas varios sobre los que nos será imposible profundizar en esta ocasión -didáctico, encomiástico, panfletario o práctico-profesional, fundamentalmente-, si bien enumeramos los ítems correspondientes en nota al pie $^{6}$.

RM 493.5, «La contienda que ovieron Ajas Telamon y Ulixes [...]. Trasladada de principio del decimo tercio libro del Ovidio», cuyo final reza "La qual embió el licenciado Alonso Rodríguez de Tudela al illustre e muy magnífico señor el señor don Hernando Enrríquez [...] para en que lean sus hijos [...]» (1519). Todas las dataciones cronológicas que se ofrecen para estos y otros impresos a lo largo del estudio, así como las adscripciones tipobibliográficas -que daremos cuando sean relevantes- se encuentran recogidas en el Nuevo Diccionario y su Suplemento.

${ }^{5}$ Por cuestiones de espacio, nos es imposible ofrecer un apéndice final que recoja el conjunto de impresos aislado para el estudio, no obstante, de la totalidad de los cuarenta y dos pliegos considerados se dará noticia de una u otra manera a través de las páginas de este trabajo, bien en cuerpo de texto, bien en nota al pie.

${ }^{6}$ Se trata de dos diálogos de adscripción didáctico-moral: Bias contra Fortuna de Santillana, con el duque de Alba como destinatario (RM 280[+281]-284), y la Murmuración de vicios de Antonio de Segovia, dirigida al prior de la iglesia mayor de Valladolid (RM 547), más, dentro del apartado didáctico, Los siete sabios de Grecia de López de Yanguas, texto ofrecido a Gabriel de la Cueva, hijo del duque del Albuquerque (RM 301.5-304.6). Junto con ellos, hallamos varios pliegos de tipo encomiástico: las Julianas de Hernando Merino, dedicadas a Julián de Medicis (RM 362), el Triunfo raymundino de Juan de Trasmiera, presentado al licenciado Luis Çapata (RM 603), el Panegírico de don Álvaro de Bazán por el licenciado Cristóbal Mosquera de Figueroa (393.5), la transmisión exenta del Dechado a la reina Isabel de Íñigo de Mendoza (RM 360), la Rima en honor del obispo don Julián de Alba (RM 77.5), de Álvaro de Cadabal Valladares de Sotomayor, autor que retomaremos en relación 
Desde una perspectiva de género, nos parece sumamente interesante que más de una quinta parte de estos treinta y ocho pliegos (21\%) tenga a una mujer como dedicataria, ocho exactamente, porcentaje que se dispara entre los de carácter religioso, llegando a casi un tercio $(31,2 \%)$ del subconjunto -cinco de dieciséis-, si bien hallamos también presencia femenina entre los destinatarios de entradas reales -así en RM 3.5, Relación de la entrada de Felipe II en Zaragoza dirigida a la Marquesa de Camarasa-, o en los Sonetos que Balbi de Correggio ofrece a Margarita de Austria con ocasión de su matrimonio (RM 48.8), impreso este último que casa con la importante presencia del rango real entre nuestras destinatarias -en cinco impresos de ocho ${ }^{7}$.

Crucial resulta destacar que ese $21 \%$ de dedicatarias identificado en el conjunto supera amplísimamente el 3,6\% de destinatarias computado por García Aguilar (2009) sobre 200 poemarios áureos pertenecientes al periodo 1543-1646, así como al porcentaje ofrecido por Nieves Baranda $(4,5 \%)$ a partir de un corpus de 131 obras publicadas entre 1500 y 1599 , si bien esta última cifra hay que tomarla con cautela en su valor contrastivo, pues Baranda (2011: 21) advierte de la no inclusión, por razones

con un segundo impreso ligado con su nombre (RM 77.3), y tres pliegos laudatorios de Francisco Balbi de Corregio, sobre los que también volveremos (RM 48.3, RM 48.5, y RM 48.8). Entran en el abanico, asimismo, las difundidísimas Coplas de Mingo Revulgo, dirigidas al Conde de Haro (RM 450.3-459.9 bis) y la Invectiva contra el heresiarca Luthero, de fray Cristóbal Mansilla, dedicada al Conde de Feria (RM 335.5); más dos impresos de carácter práctico-profesional: el Regimiento de salud, de Alonso de Zamora, ofrecido al obispo de Coria (RM 646) y el Arte para conservar el dinero en la bolsa de Gregorio Méndez, dedicado al protomédico Nicolás de Soto (RM 357 y 358). A ellos hay que sumar asuntos más variados: el pliego de carácter amoroso con la Glosa de Pedro de Aguilar dirigido a don Jorge de Silva (RM 4) y el impreso teatral Égloga interlocutoria de Diego Guillén de Ávila dedicada al Gran Capitán (RM 240.5) -texto dramático incluido en el Nuevo Diccionario solo a partir de los versos finales que rematan el pliego-.

${ }^{7}$ Al pliego de Balbi de Correggio hay que añadir la ya mencionada edición exenta del Dechado a la reina Isabel, de Íñigo de Mendoza (RM 360), pliego actualmente desconocido del que tenemos noticia por una compra realizada por Hernando Colón el 23 de noviembre de 1524 (Regestrum, n. ${ }^{\circ}$ 4108); más RM 536, Reglas del Sexto de las Decretales arromanzadas por Pedro Luis Sanz para Germana de Foix; RM 242.5-242.8, Glosa de las Coplas de Manrique por Francisco de Guzmán dirigida a Leonor de Francia y RM 381, Exposición moral sobre el salmo $L X X X V I$ de David «dirigido a la muy alta y muy poderosa señora la infanta doña María por George de Montemayor». A estas tres últimas obras habremos de referirnos nuevamente en relación con el estudio de los pliegos religiosos y moralizantes. 
metodológicas, de destinatarias reales en su estudio 8 . Este $21 \%$ se encuentra aún lejos, por otra parte, del 12\% de dedicatarias computado por Collantes y García Aguilar (2015) para el Bajo Barroco a partir de 307 obras poéticas impresas entre 1650 y 1750, incluyendo también en su caso destinatarias reales, de las que ofrecen, como para nuestro corpus, una elevada cifra (Collantes y García Aguilar, 2015: 53); por lo demás, no hay que olvidar en relación con el 12\% de Collantes y García Aguilar que, además de hablarnos del claro incremento de la presencia de la mujer en la cultura del libro a medida que avanza el Siglo de Oro, está recogiendo también dedicatorias a figuras simbólicas como la de la Virgen María9.

A la luz de los datos mostrados, y sin perder de vista que ninguno de los estudios tomados como punto de referencia atendió específicamente a pliegos poéticos -si bien en la nómina ofrecida por Collantes y García Aguilar (2015: 58-62) se recoge alguno-, el 21\% de dedicatarias acotado para nuestro conjunto resulta enormemente significativo a la hora de extraer conclusiones que habrían de llevarnos no tanto a la mayor vinculación entre figura femenina y este tipo de impresos «menores» como a la especial simbiosis entre opúsculo religioso y mujer, si es que atendemos a los porcentajes aportados sobre esta temática en particular, hecho especialmente ostensible en relación con ciertos subgéneros religiosos. En este sentido, no creemos en absoluto casualidad, más allá de lo exiguo de los datos, el que dos de los tres textos del corpus que se articulan en torno al subgénero del contemptu mundi se hallen dirigidos a mujeres, y, más concretamente, a mujeres no de vinculación regia sino nobiliaria, circunstancia que, sin duda, responde a su papel de transmisoras, dentro de la comunidad, de valores morales y sociales, según se ha insistido recientemente en trabajos como el de Baranda (2011: 35) ${ }^{10}$.

\footnotetext{
${ }^{8}$ Recuerdan Collantes y García Aguilar (2015: 51, n. 10) estos porcentajes a partir de los trabajos de Baranda (2011) y García Aguilar (2009).

${ }^{9}$ Cabe señalar que la cifra de Collantes y García Aguilar resulta más próxima a la ofrecida por Baranda (2011: 21) a partir del rastreo de la Bibliografía de la literatura hispánica de Simón Díaz: «Although it is difficult if not impossible to establish how many books were dedicated to women in comparison with men, the search I have conducted of the 14 volumes of Simón Díaz's Bibliografia de la literatura hispánica leads me to believe that the ratio could be around $10 \%$.

${ }^{10}$ Son estos tres pliegos: RM 18, Contemptus mundi dirigido por Garci Álvarez, capellán de Germana de Foix, a Fernando de Aragón (¿c. 1535?); RM 218, Menosprecio del mundo de Miguel Gilberte de Majarrés dedicado a Joana de la Caballería, señora de Alcaraz,
} 
Si abordamos desde un punto de vista material la totalidad de pliegos de tipo religioso-doctrinal y moralizante aquí estudiados, habremos de destacar, junto con su principal ubicación cronológica en la primera mitad del s. XVI -circunstancia sobre la que volveremos-, una idiosincrática codificación formal extensible a la mayor parte de los impresos que nos ocupan -dejando al margen los de carácter celebrativo y noticiero- que se caracteriza por: a) extensión que oscila, generalmente, entre las ocho y las veinte hojas -con predominio de las ocho o doce hojas-, muy superior, por tanto, a la del prototípico pliego popular de cuatro hojas; b) presencia de portada, que contraviene inmediatamente la economía de papel propia del producto editorial del pliego; c) inclusión de dedicatoria en prosa donde se reproducen los tópicos propios de este tipo de paratexto, con alusión, en ocasiones, a fuentes bíblicas y clásicas que separan al impreso todo lo ponderable de la categoría de "popular»; d) ilustración, con cierta frecuencia, a través de escudo real o nobiliario que remite de inmediato al destinatario; $y$, por fin, e) presencia, relativamente habitual también, de colofón, un elemento extraño, igualmente, al pliego poético más común en la primera mitad del siglo XVI y que, junto con la totalidad de rasgos anteriormente mencionados, hermana nuestros impresos antes con el opúsculo de ascendencia libresca que con la tradición más genuina del pliego de cordel.

Temáticamente, cabe distinguir dentro de este conjunto de pliegos religiosos dos grupos: el de los que básicamente acogen una única obra de carácter doctrinal o moralizante -once-, y un segundo grupo -bastante más heterogéneo que el anterior-donde propiamente se incluye un conjunto de poemas religiosos, vinculados o no con los ciclos litúrgicos -cinco-. Dentro del primer grupo ocupa un lugar propio el mencionado subgénero del contemptu mundi, siendo especialmente relevante, desde el punto de vista de la asimilación por el mercado de cordel de estos pliegos con dedicatoria, la vida en sueltas del Desprecio del mundo que RM 545 atribuye a un tal fray Antonio Sarmiento ${ }^{11}$. Reza la rúbrica de RM

«mi señora» (c. 1535); y RM 545, Desprecio del mundo de Fray Antonio Sarmiento, para Ana Sarmiento, condesa de Ribagorza (1546), impreso este último del que se da detenida cuenta más adelante.

${ }^{11}$ Incluido como obra de autoría propia por el dominico fray Antonio de Espinosa entre las que acompañan a sus Reglas de bien vivir (Burgos, Juan de Junta, 1552). Sobre la cuestión de la autoría, véase Cátedra (2006: 80-81). 
545: «Desprecio del mundo, compuesto por el muy reverendo y doctíssimo padre fray Antonio Sarmiento, maestro en sacra theología, de la orden de los predicadores. Dirigido a la muy illustre y escogida señora la señora doña Ana Sarmiento, condessa de Ribagorça. Con una obra de la Magdalena. 1546», con colofón que lo adscribe a las prensas zaragozanas de Diego Hernández y una estructura que responde a la comentada extensión de ocho hojas, más portada que refleja el escudo de la orden de los predicadores, en este caso, y dedicatoria en prosa. Frente a él, nos hallamos con la transmisión anónima del texto en el pliego de cuatro hojas RM 543, «Desprecio del mundo o por otro nombre llamado Espejo en quien se vean los males y defectos del mundo», donde se ha suprimido esa dedicatoria, y, al mismo tiempo, abreviado el texto, careciendo, por otra parte, de colofón que nos permita dilucidar su fecha de publicación. Si bien para este segundo pliego aventuró Rodríguez-Moñino su adscripción a los años 30, Cátedra (2006: 81) aboga por retrasar esas fechas sustentándose, justamente, «en la abreviación que exige el nuevo continente [de la literatura de cordel] en cuatro hojas, privilegiando ya un determinado formato». Por lo demás, vacilación similar con respecto a la incorporación o no de epístola dedicatoria es la que puede observarse, por ejemplo, en las tres impresiones conservadas de la Glosa peregrina de Luis de Aranda, dato que interpretamos, al hilo de lo aquí expuesto, en consonancia con lo forzado que habría de ser esta clase de paratextos para los cauces más idiosincráticos de la literatura de cordel $^{12}$.

En conexión con el desarrollo a lo largo de la centuria de vertiente tan ligada al ámbito de la literatura popular como es la de la literatura doctrinal y moralizante, cabe insistir en que la inmensa mayoría de los impresos con dedicatoria que la transmiten pertenecen a la primera mitad

${ }^{12}$ No obstante, a partir de los impresos conservados de la Glosa, la cronología no puede considerarse factor determinante para la presencia o no de la Epístola dedicatoria, pues carece de ella el primero de los testimonios conocidos, RM 25, con fecha de impresión de 1540 (Sevilla, Simón Carpintero), si bien se trata claramente de una re-edición - [los romances] ahora en esta impresión corregida y emendada de muchos errores y vicios que de antes solían tener»-, frente su inclusión en RM 25.5 y RM 26, pliegos salidos ambos de las prensas valencianas de Joan Navarro en 1561 y 1566, respectivamente. Hemos de tener en cuenta, por otra parte, que la dedicatoria nunca fue parte inseparable de una obra, hecho que Simón Díaz (2000: 142) recuerda señalando que «cuando [la obra] tenía éxito y era reeditada con frecuencia la sustituían por otras, ya el autor, ya el costeador o el impresor». 
de siglo. De hecho, a esas fechas se adscriben todos los pliegos con dedicatoria ligados al tópico del contemptu mundi localizados, situándonos, a final de siglo, ante expresiones de la materia no solo desligadas ya de destinatario individual alguno, sino de corte bastante más popular y halo contrarreformista ${ }^{13}$.

$\mathrm{Al}$ lado de los pliegos correspondientes al subgénero del contemptu mundi, un segundo subgrupo claramente aislable entre los impresos religiosos con dedicatoria y portadores de una única obra doctrinal o moralizante es el conformado por aquellos que glosan las archiconocidas Coplas de Jorge Manrique a la muerte de su padre. Entre estos se incluyen la Glosa de Alonso de Cervantes (RM 127-141.5), la Glosa de Jorge de Montemayor (RM 382 y 383) y la Glosa de Francisco de Guzmán dirigida a doña Leonor, reina de Francia (RM 242.5 y RM 242.8), cuya transmisión anónima en RM 242.8 habría de relacionarse, una vez más, con el proceso de popularización del texto ${ }^{14}$.

Particularmente es destacable, por su larguísima vida editorial, la «Glosa famosíssima sobre las coplas de don Jorge Manrique» de Alonso de Cervantes, opúsculo de veinte hojas dirigido a don Álvaro de Zúñiga, duque de Béjar, cuyo escudo queda plasmado en la portada. A él dedica Alonso de Cervantes un amplio prólogo con el que intenta volver a ganarse su favor, tras la caída en desgracia que le llevó al destierro en Portugal, prólogo que, no obstante, se pierde en al menos una de las ediciones conservadas, proceso que hemos visto ya repetido en el mercado del

${ }^{13}$ Así RM 266, articulado en torno a una particular danza de la muerte: «Desengaño de los estados, sobre el villancico de Perdone vuestra merced, en el qual se da a entender a qualquier hombre viviente que si no viviere como christiano y no hiziere penitencia de sus pecados, no terná entrada en el cielo. Hecho por Francisco de Ledesma. Visto y examinado con licencia impresso en Barcelona, en casa de Grabiel Graells y Giraldo Dotil, año de MDLXXXXIX».

${ }^{14}$ Sobre la Glosa de Guzmán, téngase en cuenta que quedó inicialmente excluida por Rodríguez-Moñino (1970), al constar, en las ediciones conocidas, de 22 hojas y sobrepasar, por tanto, la extensión de 20 que Rodríguez-Moñino se había fijado como límite para la entrada de impresos en su catálogo; sin embargo, Askins e Infantes, en su ampliación del Diccionario de Rodríguez-Moñino, deciden incorporarla, indicando en nota el asiento 242.5 que «este pliego en su edición completa parece constar de 22 hojas, lo que lo excluyó del Diccionario; no obstante, dada la complejidad editorial del mismo, hemos optado por incluir la entrada correspondiente sin más consideraciones». 
pliego $^{15}$. Hemos de añadir que, tras su primera impresión en Lisboa en 1501 , el texto contó con hasta diecisiete ediciones conocidas ${ }^{16}$, lo que es claro indicio del éxito de una composición que obviamente supera con creces las circunstancias concretas de su gestación ${ }^{17}$.

En cuanto a la Glosa de Jorge de Montemayor, es la correspondiente al texto que en RM 383 lleva por rúbrica «Glosa de diez coplas de don Jorge de Manrique hecha por Jorge de Montemayor sobre la muerte de la muy alta princesa doña María, hija del Rey don Juan tercero de Portugal, dirigidas al muy magnífico y discreto señor Juan da Silva regidor de Portugal ${ }^{18}$. Si tenemos en cuenta que la totalidad de piezas de Montemayor transmitidas en pliegos han llegado hasta nosotros bajo el manto del dedicatario nobiliario o real -a los Duques de Nájera se presenta su Cancionero de las obras de devoción en ocho hojas (1552) (RM 380.5), mientras que la Exposición moral sobre el salmo LXXXVI de David se dirige a la infanta María (1548) (RM 381)- inmediatamente hemos de concluir, además de la intervención directa de Montemayor en el proceso de edición, el importante usufructo que el portugués supo realizar no solo de sus obras mayores, sino también menores para el establecimiento y afianzamiento de vínculos sociales. No sería el único caso, de la misma manera todos los pliegos poéticos en castellano de un Francisco Balbi de Correggio, de marcados contenidos político-encomiásticos y con evidente función de promoción personal de su autor, se dirigen a un alto destinatario ${ }^{19}$.

De carácter más doctrinal que moralizante son dos impresos cuyos contenidos doctrinales se ligan en ambos casos, significativamente, con un destinatario regio: RM 225, Triunfo de la Inmaculada Concepción de Nuestra Señora (anterior a 1522), del reverendo Juan Gomeç, presentado

${ }^{15}$ Esa pérdida del prólogo ocurre en RM 128.5, impreso salido de las prensas valencianas de Jorge Costilla, c. 1511-1516.

${ }^{16}$ Es este el número de ediciones que inventaría Fernández Valladares en Fernández Valladares, Puerto Moro y Mahiques Climent (2020).

${ }^{17}$ La obra ha sido estudiada en profundidad por Grande Quejigo (2007).

${ }^{18}$ Desconocemos la rúbrica del segundo pliego que transmite esta obra, RM 382, por conservarse falto de portada.

${ }^{19}$ A los ya mencionados Sonetos ofrecidos a Margarita de Austria por su matrimonio (RM 48.8), hay que añadir RM 48.3, Sonetos dirigidos a Juan de Austria (1574), y RM 48.5, «En la muerte del sereníssimo duque Ottavio Farnes, duque de Parma [...]. Al sereníssimo Prínçipe su nieto, Ranucio Farnes, prínçipe di Parma, y Plazençia, mi señor, \&c.» (c. 1586). 
al infante don Enrique de Aragón y Sicilia, en conexión con la tradicional defensa de la tesis inmaculista en la Corona de Aragón ${ }^{20}$, y RM 536, Reglas del Sexto de las Decretales arromanzadas por Pedro Luis Sanz para la reina Germana de Foix (1535). No es superfluo comentar que una y otra obra se publican "con privilegio»-tampoco son las únicas en nuestro corpus-, control sobre la impresión que resulta muy ajeno al ámbito del genuino pliego de cordel, particularmente en la primera mitad del siglo XVI, y que nos asoma, una vez más, a la estrecha relación de estos impresos con el universo del libro.

Cierran el primer subgrupo de opúsculos de vinculación religiosa y moralizante dos obras más: el Despertador de pecadores (c. 1525 en su primera edición), dirigido al Almirante de Castilla Fadrique Enríquez de Cabrera (RM 837.5-840 [+841]), poema de carácter alegórico-moral de transmisión anónima en todos los testimonios conservados, una circunstancia que sin duda se relaciona, en este caso, con su fuerte carga invectiva y de crítica social ${ }^{21}$. A él se une, por fin, la Ensalada de metros que contiene los siete vicios capitales (1552) (RM 337.5), dedicada al señor de Luque, Pedro de Venegas, por un clérigo de la misma villa de Luque, lo que nos sitúa a todas luces ante una obvia estrategia de engarce dentro de la comunidad de texto que se adscribe a la tradición de los Siete pecados mortales de Mena y sus continuadores, de amplia difusión en plie$\operatorname{gos}^{22}$.

Por lo que se refiere al segundo grupo de impresos de carácter religioso, los que incluyen un conjunto de poemas devotos, ligados frecuentemente a los textos sagrados, constituyen un pequeño subcorpus bastante más variado que el anterior. En él habremos de añadir a los citados Cancionero de obras de devoción de Montemayor o Glosa peregrina de Aranda, una justa impresa en 1542 en loor de San Sebastián y San Isidro, presentada al Duque de Medina Sidonia como protector de la cofradía de

${ }^{20}$ El pliego ha de ser anterior al año 1522, año en que muere el infante Enrique de Aragón y Pimentel. Ha sido parcialmente estudiado por Carro Carbajal (2008: 537-541), quien comenta la probable condición de franciscano de su autor.

${ }^{21}$ Es texto por el que muy recientemente se ha interesado Gamba Corradine (2018) en relación con la difusión de la invectiva anti-luterana en España, y del que afirma preparar estudio específico.

${ }^{22}$ De este impreso dio noticia Fernández Valladares (1999), quien lo describe y analiza ampliamente. 
nuestra Señora de la Consolación (RM 910bis), en vinculación con el peso propio del género de la justa religiosa entre los pliegos poéticos ${ }^{23}$. Igualmente, forman parte de este grupo dos impresos de la segunda mitad del siglo XVI de ciertas características comunes que apuntan hacia su asimilación con la codificación editorial del pliego popular de cuatro hojas, ello, desde la limitación de su extensión a ese número de hojas hasta la ausencia de prólogo dedicatorio -si bien el segundo impreso presenta un soneto proemial-. En ambos casos es una autoridad eclesiástica la destinataria de versos conectados con los textos bíblicos, anónimos en el primer pliego, el que, sin duda, es de factura más popular: hablamos de RM 696.5, «Tres romances y muchos villancicos, sacados de la sagrada escriptura [...] endereçados / al muy reuerendo señor Diego de Lovera, canónigo de Salamanca» (1557); y RM 264, «Al illustríssimo y reverendíssimo señor don Juan Martínez Siliceo, arçobispo de Toledo [...]. Alvorada trobada por don Juan Hurtado, vecino de Madrid, en doze sonetos castellanos, con sus desechas o tornadas, a reverencia del nacimiento de Nuestro Señor» (segunda mitad del s. XVI) ${ }^{24}$.

Según adelantábamos, no nos es posible profundizar aquí en otras temáticas de menor representación dentro del corpus acotado, brevemente apuntadas al inicio de este estudio, no obstante, sí se hace necesario comentar de manera particular el conjunto de pliegos con destinatario que conecta con la relación noticiera. Entre estos, destaca el subgrupo correspondiente a entradas reales en una ciudad, relacionado con la importante presencia del acontecimiento regio en el pliego de noticias. Concretamente, la narración de entradas reales la localizamos hasta en cuatro pliegos -de cinco noticieros ${ }^{25}$-, en los que se nos remite al recibimiento en Zaragoza, Barcelona, Badajoz o Salamanca de Felipe II, Carlos V o Sebastián I de Portugal ${ }^{26}$. Por contraste con lo que ocurre con varios

${ }^{23}$ Baste recordar, a este propósito, que los asientos RM 907-910bis del Nuevo Diccionario se corresponden con justas literarias de carácter religioso.

${ }^{24}$ Es impreso no datado, pero claramente perteneciente a la segunda mitad del s. XVI desde su letrería redonda misma, inhabitual para el mercado del pliego en fechas anteriores.

${ }^{25}$ Dentro de nuestro corpus, a los cuatro pliegos con entradas reales que nos ocuparán a continuación hay que sumar, en su vinculación noticiera con el acontecimiento real, la "Obra hecha sobre la muerte de la sereníssima reyna de España nuestra señora doña Isabel de la Paz. Compuesta por Alonso de Ávila Capilla. Dirigida al Illustríssimo señor don Luis Pimentel y de Herrera, conde de Mayorga» (RM 42).

${ }^{26}$ Se trata del pliego ya referido RM 3.5, Relación de la entrada de Felipe II en Zaragoza 
de los autores ligados a los impresos anteriormente considerados, ninguno de los responsables de estos textos celebrativos ha pasado a nuestra historia literaria; sin embargo, de forma relevante, hasta tres de ellos se dirigen al corregidor de la correspondiente ciudad, lo que claramente nos aporta un transcendente dato sobre el tejido de redes políticas y sociales dentro de las urbes modernas. Por otra parte, su predominante extensión de cuatro hojas -así en el homogéneo subgrupo de los tres textos dirigidos a corregidores ${ }^{27}$, , asimilable al pliego popular, junto con la común ausencia de prólogo, nos sitúa de inmediato ante una tradición divergente de la del opúsculo de filiación libresca que mayoritariamente nos ha ocupado en páginas anteriores para enlazar, antes bien, con el formato propio de la relación en verso, de conexión umbilical con la carta de nuevas y su consustancial destinatario, a la que responden varios impresos del Nuevo Diccionario de los que ya advertimos su no inclusión en el corpus delimitado.

En cuanto a los cuatro pliegos de características muy especiales aludidos al comienzo de este trabajo y considerados diferenciadamente, nos encontramos aquí, en primer lugar, con el impreso de tipo burlesco «Pro-

(1553) dirigido a la Marquesa de Camarasa, «su señora», por Julián de Aguilar; más RM 481-481.5, «Tratado nuevamente hecho en metro castellano en loor y alabança del emperador y emperatriz nuestros señores, contando las grandezas y hazañas que ha hecho e haze, haziendo memoria de la forma y manera como fue entrado en Barcelona, y de las grandes fiestas que en sus reynos y señoríos se hazen por su victoriosa venida. Dirigido al illustre e muy magnífico señor don Pedro de Navarra Marichal, corregidor de Toledo. Hechas por Juan de Revenga»; RM 496, «Famossísimos romances. El primero trata de la venida a Castilla del muy alto y muy poderoso señor don Sebastián primero deste nombre, rey de Portugal, y del rescebimiento que la muy illustre y muy leal ciudad de Badajoz hizo a su alteza por mandado de su magestad. Repartido en tres cantos. El segundo y tercero tratan de la solemnidad con que fue recebido a la puerta de Sancta Marina, y cómo fue llevado por las calles principales desta ciudad. Y de la libertad que se dio a los presos que no tenían parte contraria. Compuestos por Joachin de Cepeda, natural de Badajoz. Dirigidos al illustre señor el licenciado Diego de Hoyo, corregidor y justicia mayor en la dicha ciudad y su tierra»; y RM 540, "Las obras siguientes hizo G[¿abri?]el de Sarabia en loor de nu[ciestro?] alto emperador con otra obra [iं?] muy alegre y desseada venid[ $\dot{i} a$ ?] con sus villancicos y cancion[¿es?]. Dirigió esta obra el autor [¿al?] muy manífico señor don J[¿u?] $a n$ Manrrique de Luna, corregidor en la [¿ciu-?]dad de Salamanca. Revistas y con licencia impresas. [Al fin:] Impressas en $\mathrm{Sa}$ [lamanca]. En casa de Castañed[a]».

${ }^{27}$ Difiere de este subgrupo la única relación de entrada real de destinatario femenino, la repetidamente nombrada Relación de la entrada de Felipe II en Zaragoza dirigida a la Marquesa de Camarasa (RM 3.5), impreso de 8 hojas que recoge, en realidad, una relación en prosa a la que sigue una «Recitación de Aragón» en verso. 
nósticos o juyzios astrologales suptilíssimos y verdaderos de casos estupendos y estrañíssimos, los quales se verán Deo volente en este año 1593. Traduzidos de lengua úngara en metro español por el muy docto y grande astrólogo maestre Rodolpho Stampurch, natural de la ciudad de Brida, y vezino de su calle. Dirigidos al muy invicto y muy poderoso señor Ladislao Sterlinch, pretensor de los Reynos Equinoctiales del viejo mundo» (RM 551), cuya grandilocuente dedicatoria, trufada de lugares comunes en clave paródica, resulta buen testimonio de la tipificación de la práctica y sus convenciones a finales del s. $\mathrm{XVI}^{28}$.

Particularmente interesantes -y peculiares- son los impresos registrados en el Nuevo Diccionario que nos han transmitido de manera exenta la dedicatoria que acompañaba a un volumen mayor. A relevantes estrategias editoriales orientadas a la promoción de una obra nos conduce el pliego que tiene por rúbrica «Breve parlamento que hizo el orador y poeta Álvaro de Cadabal Valladares de Sotomayor, al mui alto y poderoso señor don Sebastián, rey de Portogal [...] quando le offresció la Paráphrasis que compuso sobre el quarto Libro de Antonio de Nebrissa, cerca de la construçión de las partes de la Oraçión, con una salutaçión a Nuestra Señora» (RM 77.3), publicación en romance impresa en el mismo año (1565) y taller (Francisco Correa) que el libro en latín al que se vincula, In librum quartum Antonii Nebrissensis, y a la que rematan, además de los versos a Nuestra Señora aludidos en el encabezamiento, una glosa de «Tiempo bueno ${ }^{29}$. A su lado podrían mencionarse-con reservas-dos «pliegos» de Díaz Tanco de Fregenal, «Terno dialogal autual de Vasco Díaz Tanco de Frexenal dedicado al ilustríssimo señor don Pedro de Velasco, condestable de Castilla, conde de Haro e Duque de Frías ec.» (RM 168.3) y «Terno farssario autual de Vasco Díaz Tanco de Frexenal, endereçado al illustre e cathólico señor don Juan Puertocarrero, marqués de Villanueva del Fresno e de Moguer señor ec.» (RM 168.5), sin que se nos hayan conservado los volúmenes a los que acompañarían, si es que llegaron a publicarse, siendo bastante complejo, de hecho, el estatuto editorial de estos

\footnotetext{
${ }^{28}$ El pliego es pormenorizadamente estudiado por Hurtado y García de Enterría (1981).

${ }^{29}$ Los dos impresos son recogidos por Díaz y Díaz (1996: 345-346) en su relación de obras de Cadabal.
} 
dos impresos, pese a hallarse inventariados como pliegos sueltos poéticos en el Nuevo Diccionario ${ }^{30}$.

Por la meridiana claridad del ejemplo, especialmente elocuente nos resulta el mencionado pliego de Cadaval a la hora de considerar el aparato de publicidad vinculado con un libro en concreto y el papel que nuestros impresos «menores» podían cumplir en ese sentido, línea de estudio a la que ya se asomó, desde laderas diferentes, Baranda (1985: 21-25) al analizar conexiones entre la salida al mercado de un determinado relato caballeresco e impresión en sueltas de su correspondiente romance. La asimilación del Breve parlamento de Cadaval con una dedicatoria exenta es más que significativa, por otra parte, para valorar tanto la entidad propia que pueden llegar a adquirir estos paratextos como las dimensiones reales de su peso específico en cuanto presentación, aval y promoción de una obra a la que, ante todo, se posiciona socialmente, adelantándose, así, de alguna manera, el discurso social al literario, sin que uno y otro puedan desvincularse.

A partir del análisis expuesto en las páginas precedentes se hace necesario insistir, en primer lugar, sobre la innegable heterogeneidad del conjunto de pliegos sueltos poéticos del siglo XVI. En él es diferenciable un subgrupo muy idiosincrático y fácilmente reconocible desde su habitual codificación editorial misma: aquel que incluye un dedicatario de alto rango, elemento de proyección social que ha de hacernos reflexionar, inmediatamente, sobre el marbete de literatura "popular» asociado de forma genérica con estos impresos, así como sobre su grado de consideración y prestigio. Al tiempo, la aprehensión del conjunto desde una perspectiva de género nos ha permitido profundizar en el papel de la mujer en el entramado de la literatura del Siglo de Oro, siendo constatable en nuestro corpus un clarísimo incremento del número de destinatarias con respecto a los porcentajes ofrecidos por otros estudios atentos a la vinculación entre textos áureos y dedicatarias, sin que sea posible desvincular este dato, por otra parte, de la importantísima presencia de la temática

${ }^{30}$ Ambos impresos procederían de las prensas zaragozanas de Pedro Hardouin, c. 15281532. Vid. la amplia nota que García-Bermejo (1996: 90) les dedica, donde recoge la hipótesis de Salvá de que las obras a las que acompañarían no habrían llegado a imprimirse, comúnmente admitida. 
religiosa en el conjunto acotado, lo que nos ha permitido corroborar la especial conexión entre tal materia y mujer, puesta ya de manifiesto por otros trabajos. De la misma manera, hemos comprobado el lugar específico ocupado por el pliego poético en el tejido de redes políticas y sociales dentro de la urbe moderna desde la consideración de un segundo subgrupo temático, el de los pliegos noticieros y, más concretamente, el de los pliegos dedicados a entradas reales, común y significativamente dirigidos al corregidor de la correspondiente ciudad. Por fin, la particular atención al pliego transmisor de la dedicatoria exenta de una obra mayor ha permitido valorar en toda su profundidad el alcance de estos impresos «menores» en cuanto posibles instrumentos de publicidad y promoción para el libro áureo, dimensión esta última que viene a completar la visión aportada sobre la proyección social del pliego quinientista marcado por la presencia de dedicatario de alto rango y su particular lugar en una comprensión más cabal del universo de la literatura del Siglo de Oro.

\section{BIBLIOGRAFÍA CITADA}

ASKINS, A.L.-F. e INFANTES, V. (2014): Suplemento al Nuevo Diccionario bibliográfico de pliegos sueltos poéticos (siglo XVI) de Antonio Rodríguez-Moñino. L. Puerto Moro (ed.). Vigo, Academia del Hispanismo.

BARANDA, N. (1985): «Historia caballeresca y trama romanceril: La Historia del rey Canamory el Romance del Infante Turián». Studi Ispanici, 10, págs. 9-31.

- (2011): «Women's Reading Habits: Book Dedications to Female Patrons in Early Modern Spain». En Cruz, A.J. y Hernández, R. (eds.): Women's Literacy in Early Modern Spain and the New World. Surrey-Burlington, Ashgate, págs. 19-39.

CÁTEDRA, P.M. (2006): «Del claustro al pliego suelto: La obra de Antonio de Espinosa». En Schaffer, M. y Cortijo, A. (eds.): Medieval and Renaissance Spain and Portugal: Studies in honor of Arthur L.-F. Askins. Woobridge, Suffolk, Boydell and Brewer, págs. 68-91.

CARro CARbajal, E.B. (2008): «Concepto escolástico y concepción popular: límites de uso de la literatura religiosa en el Renacimiento». En San José, J. et al. (eds.): La fractura historiográfica: las investigaciones de Edad Media y Renacimiento desde el Tercer Milenio. Salamanca, SEMYR, págs. 533-543. 
Collantes, C.M. y García Aguilar, I. (2015): «Dedicatarias femeninas en la poesía impresa del bajo barroco». Criticón, 125, págs. 49-64.

DíAZ y DíAZ, M.C. (1996): «Álvaro de Cadaval, primer latinista de la universidad de Santiago: unas notas». Cuadernos de Estudios Gallegos, 43, fasc. 108, págs. 323-359.

Di Stefano, G. (2014): «Pliegos sueltos poéticos con destinatario declarado». $R e$ vista de poética medieval, 28, págs. 211-224.

FERNÁNDEZ VALLADARES, M. (1999): «Un pliego suelto burgalés ahora nuevamente hallado: La Ensalada de metros de Pedro Marín (1552)». Rivista di Filologia e Letterature Ispaniche, 2, págs. 95-106.

Fernández Valladares, M., Puerto Moro, L. y Mahiques Climent, J. (2020): Pliegos sueltos poéticos del siglo XVI en bibliotecas de Francia (2 vols.). México, Frente de Afirmación Hispanista (en prensa).

GAMBA CORRADINE, J. (2018): «Lutero en pliegos: hacia un corpus de pliegos castellanos quinientistas con representaciones del hereje (I)». eHumanista, 40, págs. 411-435.

García Aguilar, I. (2009): Poesía y edición en el Siglo de Oro. Madrid, Calambur. García-Bermejo, M.M. (1996): Catálogo del teatro español del siglo XVI. Salamanca, Universidad de Salamanca.

GARCÍA DE ENTERRÍA, M.C. [1973]: Sociedad y poesía de cordel en el Barroco. [Madrid], Taurus.

GRANDE QueJigo, F.J. (2007): «La "Glosa famosíssima” de Alonso de Cervantes: política y moral en la recepción de las “Coplas” de Jorge Manrique». En López Castro, A. y Cuesta Torre, M.L. (eds.): Actas del XI Congreso Internacional de la Asociación Hispánica de Literatura Medieval. León, Universidad de León, vol. 2, págs. 651-659.

Hurtado, A. y GARCíA DE ENTERRÍA, M.C. (1981): "La astrología satirizada en la poesía de cordel: El "Juyzio" de Juan del Encina y los "Pronósticos” de Rodolfo Stampurch». Revista de literatura, 43.2, págs. 21-62.

INFANTES, V. (1989): «Edición y realeza: Apuntes sobre los pliegos poéticos incunables». En Criado de Val, M. (ed.): Literatura hispánica, Reyes Católicos y Descubrimiento (Actas del Congreso Internacional sobre Literatura hispánica en la época de los Reyes Católicos y el Descubrimiento). Barcelona, PPU, págs. 85-98.

RodríGuez-MoÑIno, A. (1970): Diccionario bibliográfico de pliegos sueltos poéti$\cos ($ s. XVI). Madrid, Castalia. 
RodríGUEZ-MoÑINO, A., Askins, A.L.-F. e INFANTES, V. (1997): Nuevo Diccionario bibliográfico de pliegos sueltos poéticos (s. XVI). Madrid, Castalia-Editora Regional de Extremadura.

Simón DíAZ, J. (2000): El libro español antiguo. Madrid, Ollero \& Ramos.

Laura PUERTO MORO

Universidad Complutense de Madrid laurapuerto@pdi.ucm.es 0000-0003-3427-3165 